\section{Kutane Leishmaniose}

Zusammenfassung. Bei einer 34-jährigen türkischen Patientin wurde eine atypisch verlaufende kutane Leishmaniose mit tumoröser Wucherung und Exulzeration diagnostiziert und effektiv durch Kryochirurgie therapiert.

Cutaneous Leishmaniasis. A 34-year old Turkish women suffered from atypical cutaneous leishmaniasis. She was treated effectively by cryosurgery.

\section{Fallbericht}

\section{Anamnese}

Eine 34-jährige türkische Patientin wurde uns mit einem angeblich seit 3 Wochen schnell wachsenden schmerzhaften Tumor am Kinn rechtsseitig vorgestellt. Wie die Hauteruption vorher ausgesehen hat, konnte anamnestisch nicht eruiert werden. Eine diagnostische Einordnung war auswärts trotz Probebiopsie, die eine unspezifische eosinophile Dermatitis mit Akanthose, Hyperkeratose und Ödem des Papillarkörpers mit zahlreichen Eosinophilen und reichlichen Infiltraten aus Lymphozyten, Plasmazellen sowie gering auch Granulozyten erbrachte, nicht möglich. Anamnestisch kam es zu wiederholten Aufenthalten in der Türkei, zuletzt vor knapp einem Jahr.

\section{Hautbefund}

Zum Vorstellungszeitpunkt fand sich eine $3,5 \times 2,8 \mathrm{~cm}$ große tumoröse, schmalbasige Hautveränderung am Kinn rechtsseitig mit beginnender zentraler Exulzeration und Randwall. Die Umgebung des Herdes war entzündlich infiltriert (Abb.1). Das klinische Bild konnte unsererseits primär rein makroskopisch nicht eingeordnet werden. Differenzialdiagnostisch dachten wir an ein Granuloma pyogenicum, eine vegetierende Pyodermie und eine kutane Leishmaniose.

Akt Dermatol 2001; 27: 158-160

(c) Georg Thieme Verlag Stuttgart · New York ISSN 0340-2541

\section{Dj. Djawari, A. Alpagut}

Hautklinik Klinikum Heilbronn, Akademisches Lehrkrankenhaus der Universität Heidelberg (Direktor: Prof. Dr. med. Dj. Djawari)

Labor

BKS 30/61 mm n.W., CRP 1,6 mg/dl. Die restlichen ausgedehnten Routinelaborparameter einschließlich des Antistreptolysin- und Antistaphylolysin-Titers waren normwertig.

\section{Histologie}

Die erneute Probebiopsie zeigte lediglich eine unspezifische abszedierende Entzündung mit reichlich neutrophilen und eosinophilen Granulozyten sowie Kerntrümmer in Makrophagen.

Im Abstrichpräparat des Wundgrundes gelang uns jedoch der Nachweis von Leishmanien (Abb. 2). Kulturell wurden zusätzlich vergrünende Streptokokken nachgewiesen.

\section{Therapie und Verlauf}

Morphologisch stand zunächst das Granuloma pyogenicum mit geringer Exulzeration im Vordergrund. Nach Sicherung der Diagnose wurde der Herd trotz der tumorösen Wucherung kryochirurgisch mittels Kryosprayfreezing therapiert. Aufgrund der bakteriellen Superinfektion mit vergrünenden Streptokokken bekam die Patientin eine antibiogrammgerechte Antibiose. Lokaltherapeutisch setzten wir nach den kryochirurgischen Maßnahmen Providonjod-haltige Salbe ein. Hierunter ist es zu einer raschen Besserung des Lokalbefundes gekommen, so dass die Patientin bereits nach kurzer Zeit entlassen werden konnte. Eine Kontrolle des Befundes erfolgte dann in unserer Ambulanz, dabei wurde eine komplette Abheilung des Herdes festgestellt (Abb. 3).

\section{Diskussion}

Die Leishmaniosen stellen eine heterogene Gruppe von Infektionserkrankungen dar, die durch Protozoen des Genus Leishmania hervorgerufen werden und in weiten geografischen Regionen der Erde, vor allem im mittleren Osten, Afrika, Asien, Zentral- und Südamerika verbreitet sind [1].

Die klinischen Erscheinungsformen der kutanen Leishmaniosen hängen im Wesentlichen einerseits von der Leishmaniaspezies andererseits von dem Immunstatus des Patienten ab [2]. Übertragen wird die Erkrankung durch den Stich einer infizierten Sandmücke (Phlebotomus bzw. Lutzomyia). Sie kann 


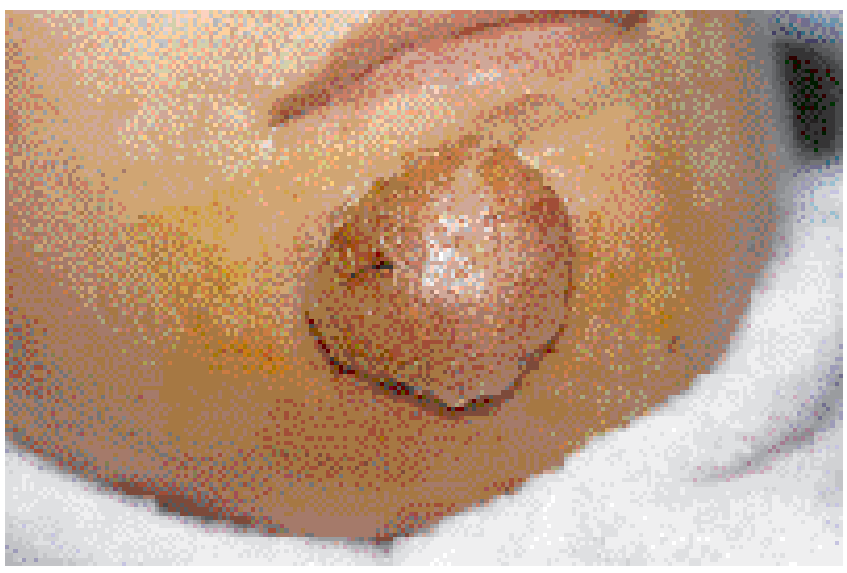

Abb.1 Tumorös wachsende kutane Leishmaniose mit Exulzeration am Kinn einer 34-jährigen Patientin.

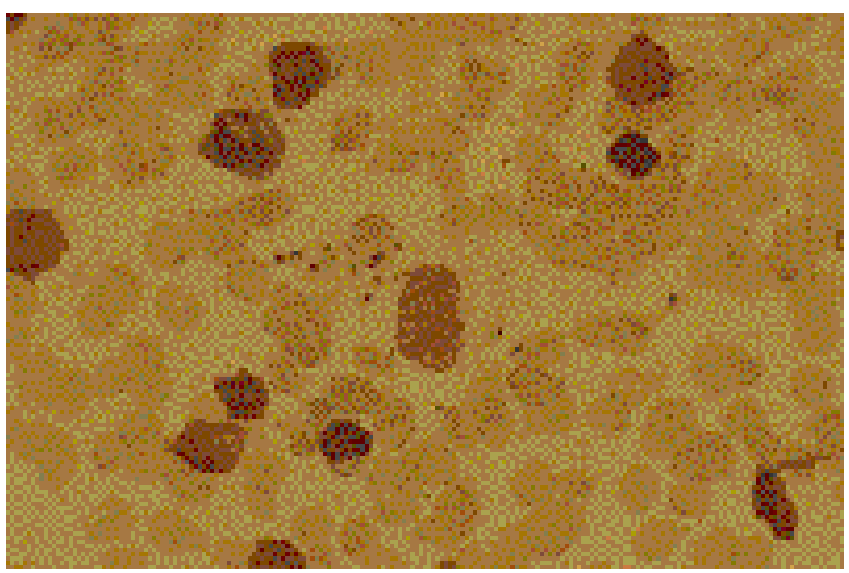

Abb. 2 Abstrichpräparat vom Wundgrund der Hautveränderung der Patientin von Abb. 1 mit intrazellulären Leishmanien.

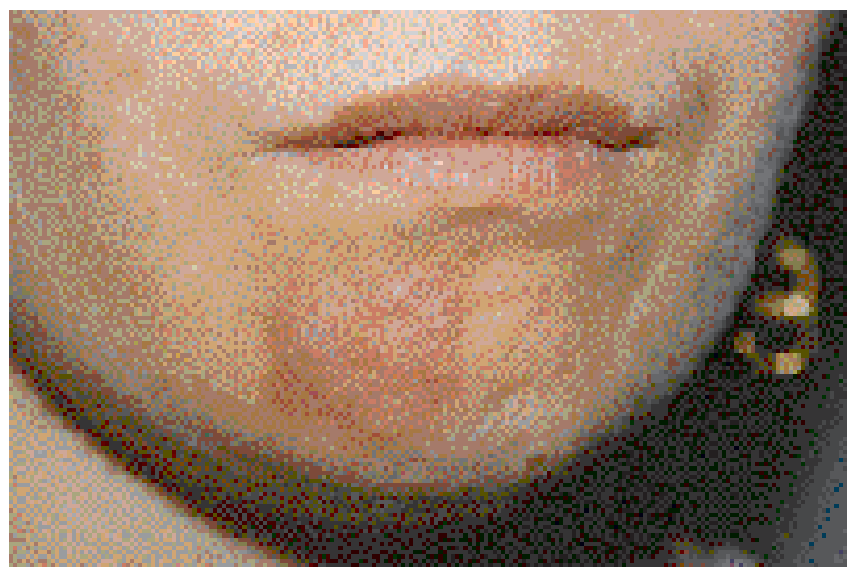

Abb. 3 Die Patientin von Abb.1 mit vollständiger Abheilung des Herdes nach kryochirurgischer Therapie. jedoch auch kongenital, sexuell oder parenteral sowie durch den direkten Kontakt übertragen werden [3].

Klinisch kann die kutane Leishmaniose lokalisiert, disseminiert, rezidivierend und auch mukokutan auftreten [1]. Hierbei ist zu unterstreichen, dass die lokalisierte Manifestation als häufigste Form der Leishmaniose, vor allem an frei exponierter Haut vorkommt [4].

Klinisch beginnt die Erkrankung meist 2-4 Wochen nach dem Insektenstich mit einer kleinen erythematösen Papel, welche im Laufe der nächsten Wochen bzw. Monate langsam wächst. Hier können sich im Verlauf Noduli, Plaques, Ulzerationen, krustös belegte, trockene oder auch teils nässende Herde ausbilden. Möglich ist das Auftreten von kleinen Satellitenknötchen periläsional und gelegentlich eine begleitende Lymphangitis sowie Lymphadenopathie. Sekundär tritt häufig eine Superinfektion auf. Die kutane Leishmaniose kann innerhalb von 3-14 Monaten spontan unter Ausbildung von hypo- bzw. hyperpigmentierten atrophen Narben abheilen. Sie kann jedoch auch 2-5 Jahre aktiv bleiben. Abhängig vom Immunstatus des Patienten können schwerste erosive und ulzerative Hauterscheinungen auftreten [3].

Differenzialdiagnostisch können Leishmaniaveränderungen knotigen bzw. granulomatösen Hautveränderungen z. B. einer kutanen Tuberkulose, einer Sporotrichose, einer atypischen Mykobakterose (Schwimmbadgranulom), der Lepra, einem Furunkel, den Mykosen, der Malaria, einer kutanen Sarkoidose, einem kutanen Lupus erythematodes, den Fremdkörpergranulomen, einem Ecthyma infectiosum Orf oder auch den kutanen Neoplasien ähneln. Bei der disseminierten Form der Erkrankung können multiple rote oder livid-rote, papulöse oder nodöse Läsionen auftreten, die den größten Teil des Integuments bedecken. Im Gegensatz dazu kann das Erscheinungsbild der rezidivierenden Form durch das Auftreten von erythematosquamösen Papeln am Rand einer Narbe gekennzeichnet sein [4]. Die mukokutane Form lässt sich durch kleine, einzelne oder auch multiple, rötliche oder livid-rote Papeln, von denen einige ulzerieren und andere zu Plaques konfluieren, diagnostizieren. 4 Monate bis 12 Jahre nach Auftreten der Veränderungen, die sich meistens spontan zurückbilden, treten die ersten Veränderungen der Schleimhäute auf, mit Bevorzugung der Nase und der Lippen. Die häufigste Prädilektionsstelle ist hier der Nasenknorpel, wo es zu Infiltration, Entzündung, Ulzeration bis zur Perforation des Nasenseptums kommen kann. Selbst Rachen, Tonsillen, Trachea, Bronchien, Augen und die Schleimhäute des Urogenitaltraktes können befallen sein [2-4].

Die Leishmaniose kann lokal und/oder systemisch behandelt werden. Die lokale Therapie der kutanen Leishmaniosen ist bei Patienten mit einzelnen Läsionen indiziert. Hier sind die chirurgische Exzision, die Kürettage, die Elektrodessikation, der $\mathrm{CO}_{2}$-Laser, die Hyperthermie, Paromomycinsulfat [5] und wie hier appliziert, die Kryochirurgie zu erwähnen. Die Kryochirurgie, welche ein einfach durchzuführendes, kostengünstiges und zudem sehr effektives Behandlungsverfahren darstellt [6], ist an erster Stelle zu empfehlen.

Systemische Therapien sind indiziert bei disseminiertem Hautbefall, multiplen großen Hautveränderungen, gleichzeitigem Schleimhaut- bzw. Lymphknotenbefall sowie bei erfolglo- 
ser Lokaltherapie. Es bieten sich hier verschiedene Therapeutika wie die fünfwertigen Antimon-Verbindungen, Pentamidin [7,8], Allopurinol [9], Ketokonazol [10] und Rifampicin oder Metronidazol an.

Das interessante bei unserem Kasus war die klinische Morphologie mit einem tumorösen schnellen Wachstum. Dieser Verlauf wurde sicherlich durch eine bakterielle Superinfektion verursacht, worauf ein Granuloma pyogenicum-artiges Wachstum aufgetreten war. Sowohl das Granuloma pyogenicum als auch die kutane Leishmaniose behandeln wir sehr effektiv durch Kryochirurgie [6].

\section{Literatur}

${ }^{1}$ Tsambaos D. Kutane Leishmaniosen. Z Hautkr 1998; 73: 725 - 733

2 Zimmermann R, Zettl H, Schlisio W, Möller FW. Leishmaniasis cutis. Akt Dermatol 1996; 22: 156-160

${ }^{3}$ Tronnier M, Wolff H. Leishmaniosen - Dermatologische Manifestationen, Diagnostik und Therapie. Z Hautkr 1996; 71: 549 553

${ }^{4}$ Jappe U, Gollnick H. Tropendermatologie, Reisemedizin, Impfungen (Teil 1). Hautarzt 1998; 49: $421-434$

${ }^{5}$ Weinrauch L, Cawich F, Craig P, El-on J, Sosa SIX. Topical treatment of New World cutaneous leishmaniasis in Belize: A clinical study. J Am Acad Dermatol 1993; 29: 443-446

${ }^{6}$ Djawari D, Barsom O. Kryochirurgie - Renaissance einer effektiven Therapie. Akt Dermatol 1993; 19: 322 - 326

${ }^{7}$ Blum J, Hatz C, Junghans T. Therapie kutaner und mukokutaner Leishmaniosen. Dtsch med Wschr 1994; 119: 1169-1172

${ }^{8}$ Koff AB, Rosen T. Treatment of cutaneous leishmaniasis. J Am Acad Dermatol 1994; 31: 693-708

${ }^{9}$ Barzilai A, Friedman J, Trau H. Treatment of cutaneous leishmaniasis with allopurinol. J Am Acad Dermatol 1995; 32: 518

${ }^{10}$ Tratner A, Ingber A, Segal B, Wiesmann-Katzenelson W, Sandbank M. Ein ungewöhnlicher Fall: Haut-Leishmaniose mit subkutanen Knoten - erfolgreich behandelt mit Ketokonazol per oral. Z Hautkr 1990; 65: 927-928

Prof. Dr. med. Dj. Djawari

Hautklinik am Gesundbrunnen

74064 Heilbronn

\section{BERICHTIGUNG}

B. M. Hausen, U. Stephan, G. Heidbreder: p-PhenylendiaminKontaktallergie als Urlaubssouvenir. Akt Dermatol 2000, 26: 230-234

Den Autoren ist leider ein Fehler unterlaufen. Auf der Seite 233, letzter Absatz, 7.Zeile: Anstatt der Formulierung „Als Haarfärbemittel ist PPD in der Bundesrepublik... verboten“ muss es richtig lauten „Als Farbstoff zur direkten Applikation auf der Haut ist PPD in der Bundesrepublik und anderen EGLändern daher seit Jahrzehnten verboten.“ 